\title{
Route-Based Signal Preemption Control of Emergency Vehicle
}

\author{
Haibo Mu (D), Yubo Song, and Linzhong Liu \\ School of Traffic and Transportation, Lanzhou Jiaotong University, Lanzhou, Gansu 730070, China \\ Correspondence should be addressed to Haibo Mu; mhbmmm@mail.lzjtu.cn
}

Received 30 October 2017; Accepted 20 February 2018; Published 1 April 2018

Academic Editor: Ai-Guo Wu

Copyright (C) 2018 Haibo Mu et al. This is an open access article distributed under the Creative Commons Attribution License, which permits unrestricted use, distribution, and reproduction in any medium, provided the original work is properly cited.

\begin{abstract}
This paper focuses on the signal preemption control of emergency vehicles (EV). A signal preemption control method based on route is proposed to reduce time delay of EV at intersections. According to the time at which EV is detected and the current phase of each intersection on the travelling route of EV, the calculation methods of the earliest start time and the latest start time of green light at each intersection are given. Consequently, the effective time range of green light at each intersection is determined in theory. A multiobjective programming model, whose objectives are the minimal residence time of EV at all intersections and the maximal passing numbers of general society vehicles, is presented. Finally, a simulation calculation is carried out. Calculation results indicate that, by adopting the signal preemption method based on route, the delay of EV is reduced and the number of society vehicles passing through the whole system is increased. The signal preemption control method of EV based on route can reduce the time delay of EV and improve the evacuation efficiency of the system.
\end{abstract}

\section{Introduction}

Once an unexpected event has happened, an evacuation or a rescue must be carried out to help people in hazardous situations, and emergency vehicles (EV) play an important role in this process. To reduce the negative impact of unexpected events, EV is used to transferr people from dangerous areas to emergency shelters or medical assistance organizations as rapidly as possible. As is well-known to us all, the quality of the emergency service relies on the travel time that EV spends in the evacuation route $[1,2]$. Therefore, EV has a greater priority than normal social vehicles, and signal preemption policy should be adopted to ensure the rapid transit of EV. However, under the condition of traffic jams or if there is no emergency lane, the queuing vehicles may prevent EV from passing through the intersection. Even if there is an emergency lane, to avoid an urgent spectacle, EV also has to wait if green traffic light for the opposite direction is going on.

To ensure that EV can pass through intersections safely and rapidly, some scholars proposed the signal preemption strategies. Paniati and Amoni [3] summarized the advantages of traffic signal preemption for EV in improving response speed, ensuring safety, saving cost, and other aspects and pointed out some of the key technologies used in traffic signal preemption system as well as some associated responsibility institutions. Mirchandani and Lucas [4] studied control strategies by integrating transit signal priority and rail/emergency preemption within a dynamic programmingbased real-time traffic adaptive signal control system. Song [5] proposed traffic signal priority control method for multiple EVs based on multiagent and obtained signal priority control strategies through the coordination of phase agent and management agent. Taking the minimum delay of EV at single intersection as control target, three signal priority strategies, namely green time extension, red time shortening, and multiphase integrated control were proposed by Yang et al. [6], and Vissim was adopted to evaluate the proposed strategies. Ma and Cui [7] studied the signal priority problem of EV that was coming from different directions and had to pass the same intersection in the certain time period and presented a signal priority control system for multiple EV based on multi-agent. Wang et al. [8] proposed a degreeof-priority based control strategy for emergency vehicle preemption operation to decrease the impacts of emergency vehicles on normal traffic, and the performance of the proposed strategy was compared with the conventional local detection based method under the microscopic simulation model. To reduce the response time and minimize the impact 
of EV operation on general traffic, Qin [9] proposed two new control strategies for EV signal preemption, where the first strategy was developed to enable signal transition from normal operation to EV signal preemption and the second control strategy was used for the transition from EV signal preemption back to normal operation.

Most of the signal preemption methods adopted currently are based on isolated intersection, namely, taking single intersection as a basis, and signal preemption of this intersection will be activated after local detector has detected EV and thus form a signal priority sequence from intersection to intersection $[10,11]$. This kind of signal preemption strategy, which is based on local detection and clears the intersection one by one, can start only after an EV has been detected and usually leads to inevitable intersection delay. Moreover, when multiple signal preemptions are implemented during peak period, the queue and delay of social vehicles in a given network will be significantly affected [12].

Studies have shown that implementing signal preemption from the perspective of entire route can reduce the response time of EV [13]. Although substantial progress has been made in signal preemption of isolated intersection, research of dynamic signal preemption based on entire route is rarely seen. Kwon and Kim [14] developed a route-based signal preemption strategy. They used Dijkstra's algorithm to obtain an optimal path from a given origin to a desired destination so as to ensure a minimal travel cost under current traffic conditions. The control algorithm chose a specific phase combination for each intersection on the selected route sequentially when EV arrived. But, the main purpose of this research was to ensure the safety of pedestrians passing through intersection, and real-time traffic conditions of various intersections were ignored. To reduce travel time of EV, Gedawy et al. [15] combined signal preemption with dynamic route planning and designed route of EV from the perspective of network. A signal preemption strategy, which could ensure the safe operation of an EV and maximize traffic flow passing through the intersection at the same time, was proposed. In order to provide green wave for EV, Kang et al. [16] proposed a coordinated signal control method and carried out traffic simulation taking eight intersections as example. All the methods mentioned above ensured the smooth running of EV by determining in advance the signal phase of each intersection after EV had been detected. Since green signal stayed at the designated phase, traffic flow from other signal phases lost right of way and they had to stay a long time at the intersection, which in turn increased the burden of traffic system and even caused traffic jams. Therefore, it is especially important to establish a routebased dynamic preemption strategy so as to provide efficient and safe operating environment for EV and minimize the interference on social vehicles.

So far, most of the developed preemption systems operate on a single intersection basis and require local detection of $\mathrm{EV}$ at each intersection to activate signal preemption sequence. Owing to the fact that these kinds of strategies depend on local detection and clear intersection one by one, and signal preemption procedure can not start until an EV is detected, inherent delays at intersections are unavoidable. The basic method of route-based signal preemption control of EV is that, when unexpected events occur, in consideration of dynamically changing traffic situations, the route that $\mathrm{EV}$ can use to reach the scene of the accident in the shortest time is calculated and recommended as the evacuation route. Once an evacuation route is selected, particular signal preemption control strategy is presented to determine the activation time for preemption at each intersection on the emergency route and realize the signal preemption control of EV ultimately.

Evacuation route planning is an important component of emergency management that seeks to minimize the loss of life or harm to the public during unexpected events [17]. The aim of evacuation route planning is to minimize evacuation time under certain constraints and minimize the possibility of depth hazards to people from accident areas. Nowadays, almost all of the intersections in urban traffic network are signalized intersections. In order to realize smooth traffic under real road condition, traffic management departments usually adopt regulation measures, including no-left-turn, no-right-turn, P-turn, and U-turn, to improve the safety and efficiency of traffic. For this reason, the road network that is connected ostensibly is not connected actually, which add difficulties to the shortest path algorithms and make some classical finding methods unavailable. Many scholars have intensively studied the shortest path problems of road network with traffic restrictions [18-23]. Various techniques have been proposed to eliminate certain turning and crossing maneuvers at intersections so as to obtain evacuation routes that can provide continuous traffic flow and reduce accidents [24-30]. There also has been a considerable amount of evacuation route works that consider delay and capacity of intersections $[31,32]$. The majority of the evacuation route works in the literature can be divided into three categories, namely, network flow methods, which can be further divided into linear programming and dynamic minimum cost flow problem [24, 33-38], simulation methods $[39,40]$, and heuristic methods, which mainly includes the well-known Capacity Constrained Route Planner (CCRP) algorithm and its improved algorithms [41-47]. The research of evacuation route planning has made substantial progress, and the development of modern detecting equipment and information technology ensures the enforceability of realtime traffic flow detection and information exchange among different intersection controllers. These technologies make it possible to implement route-based signal preemption control of EV, but a safe and efficient manner to change the traffic signal timing plan, namely, control mechanism, has not been well studied.

In this paper, we will study the problem of dynamic signal preemption control of EV based on entire route. We limit the scope of this paper to signal preemption control of EVs under the assumption that the evacuation route has been determined, and the problem of evacuation route calculation will not be discussed further. The objectives of research reported here were to (1) propose a route-based signal preemption strategy to provide efficient and safe operating environment for EV and minimize the impact on social vehicles in traffic network as far as possible, (2) develop a multiobjective optimization model to minimize the residence time of EV at 


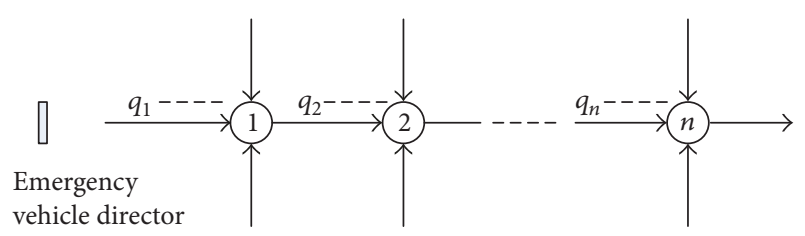

Figure 1: Diagram of intersection.

all intersections and maximize the number of social vehicles passing through all intersections, and (3) design a solving algorithm for the presented optimization model and verify the efficiency of the proposed signal preemption strategy.

First, on the premise of having selected a concrete evacuation route, taking isolated intersection as objective and considering the distance between EV detector and each intersection, the operating speed of EV and the number of queued vehicles at each intersection, the earliest-possible start time, and the latest-possible start time of green light at each intersection in the route are given. And then, in order to establish a real-time signal control strategy to ensure that EV can pass through intersection with operating speed or without stopping and minimize the impact of EV on social vehicles, a multiobjective programming model is presented and a particle swarm algorithm is designed to find the Pareto optimal solution set of this model. Finally, a simulation analysis is carried out.

\section{Calculation of Intersection Time Parameters}

After the optimal evacuation route of $\mathrm{EV}$ has been given, to ensure that the delay of EV is much smaller and the social vehicles passing through the whole system are much larger, we need to determine the most suitable time of opening the green light for EV of each intersection in the given route. Suppose that there are $n$ intersections in the evacuation route except the given origin and the desired destination, and the evacuation direction is from west to east as shown in Figure 1. What needs to be stressed here is that we just take Figure 1 as an example to introduce the following concepts and calculation methods of intersection parameters. In fact, an EV may turn left or right at some of the intersections on the given evacuation route, and the following methods are also applicable to this kind of route. We assume that EV is detected at time $t_{0}$, and $q_{i}, i=1,2, \ldots, n$ denotes vehicles waiting to pass through intersection $i$ in front of EV along the direction of evacuation at time $t_{0} ; q_{i \mathrm{~N}}, q_{i \mathrm{~S}}, q_{i \mathrm{~W}}, q_{i \mathrm{E}}, i=1,2, \ldots, n$ denotes vehicles waiting to pass through intersection $i$ in north-southward direction, south-northward direction, west-eastward direction, and east-westward direction at time $t_{0}$, respectively. Suppose that all the intersections are twophase control intersections and have fixed signal cycle, as shown in Figure 2. Each intersection $i$ corresponds to a time window sequences $W L(i)=\left(w s_{i}, w_{i 1}, w_{i 2}\right)$, where $w s_{i}$ denotes the start time of the first time window, and $w_{i k}, k=1,2$ denotes the $k$ th time window of intersection $i$.

The time parameters mentioned in this paper include the earliest-possible start time and the latest-possible start time of green light at each intersection.

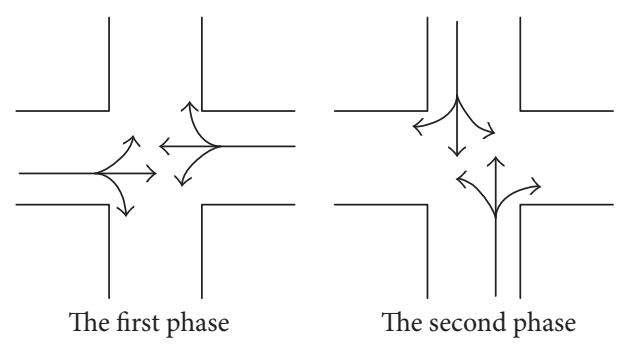

FIGURE 2: Phase diagram of traffic signal.

Definition 1. The earliest-possible start time is defined as the earliest time that traffic light of EV direction can be changed to green from the time the EV is detected and on the premise of ensuring the efficiency of the whole system.

Definition 2. The latest-possible start time is defined as the latest time that traffic light of $\mathrm{EV}$ direction must be changed to green so as to ensure that EV can pass through the intersection without reducing speed or without stopping.

2.1. Calculation of Time Parameters of Intersection 1. We employ $d_{i j}$ and $t_{0}$ to denote the duration time of phase $j$ at intersection $i$ and the time that $\mathrm{EV}$ is detected. If the starting time of the first time window of intersection 1, denoted by $w s_{1}$, is determined and the duration time of the two phases denoted by $d_{11}$ and $d_{12}$ is also determined, then the current phase of intersection 1 and the elapsed green time of this phase can be calculated correspondingly.

Provided that $C_{i}$ denotes the cycle length of intersection $i$ and $D_{i, j}$ denotes the elapsed green time of intersection $i$ after $j$ phases, the elapsed number of cycles in period $t_{0}-w s_{1}$ is denoted as $a$, and the remaining time after $a$ cycles in period $t_{0}-w s_{1}$ is denoted as $b$. Then we have

$$
\begin{aligned}
& D_{1,0}=0, \\
& D_{1, j}=\sum_{p=1}^{j} d_{1 p}, \quad j=1,2 .
\end{aligned}
$$

Let

$$
\begin{aligned}
& a=\frac{\left(t_{0}-w s_{1}\right)}{C_{i}}, \\
& b=\left(t_{0}-w s_{1}\right) \bmod C_{i}
\end{aligned}
$$

then the current phase is $i$ if it satisfies that $D_{u, i-1} \leq b<D_{u, i}$, and the elapsed green time of phase $i$ is $t_{0}-w s_{1}-a \times C_{i}-$ $\sum_{p=0}^{j-1} D_{1, p}$.

Let $G_{\min }^{j}, G_{\max }^{j}, G_{\text {ela }}^{j}$, and $G_{\text {real }}^{j}$ denote the minimal green time, the maximal green time, the elapsed green time, and the real green time of phase $j$, respectively; $L_{\mathrm{EV}-i}$ denotes the distance between $\mathrm{EV}$ detection and intersection $i$; $v_{\mathrm{EV}}, v_{\text {reg }}$ denote the speed of EV and mean speed of general traffic, respectively; $t_{\text {switch }}$ denotes the time needed to switch green indication from one phase to another phase; $t_{\text {safe }}$ is the safety time interval that must be kept between the last vehicle in 
the queue on the EV approach and the EV so as to avoid collision between EV and social vehicles. It is an important factor to ensure the safe operation of emergency vehicles and can be provided by an appropriate advance notice of the approaching of EV and a real-time traffic queue control strategy; $B$ is the average effective length of each vehicle when parking, which is equal to the sum of the length of vehicle itself and the distance between adjacent vehicles. Let $t_{E}(i)$ and $t_{L}(i) i=1,2, \ldots, n$ denote the earliest-possible start time and the latest-possible start time of phase $i$ in EV approach; then we will illustrate the calculation method of $t_{E}(i)$ and $t_{L}(i) i=1,2, \ldots, n$ beginning from the first intersection.

\subsubsection{The Calculation of $t_{E}(1)$}

(1) Phase 1 is the Current Phase of Intersection 1 at Time $t_{0}$. If the elapsed green time is $G_{\text {ela }}^{1}$ and satisfies $L_{\mathrm{EV}-1} / v_{\mathrm{EV}}+$ $G_{\text {ela }}^{1} \leq G_{\max }^{1}$, then $t_{E}(1)=t_{0}$ and the real start time of green signal at intersection 1 is $t_{0}$. Otherwise, if $L_{\mathrm{EV}-1} / v_{\mathrm{EV}}+G_{\text {ela }}^{1}>$ $G_{\max }^{1}$, then we have to determine whether $G_{\text {ela }}^{1} \geq G_{\min }^{1}$ is met at time $t_{0}$. If it is met, the green indication of phase 1 is ended and starts again after the minimal green time of pahse 2 is finished. Before this, we have to check, after phase 1 regains the green indication, whether the queuing vehicles at the intersection can be cleared before EV arrives and can maintain a certain safety interval between EV and social vehicles. If we have

$$
t_{\text {switch }}+G_{\text {min }}^{2}+t_{\text {switch }}+\frac{q_{1} \times B}{v_{\text {reg }}}+t_{\text {safe }} \leq \frac{L_{\mathrm{EV}-1}}{v_{\mathrm{EV}}}
$$

then $t_{E}(1)=t_{0}+2 t_{\text {switch }}+G_{\text {min }}^{2}$. If $G_{\text {ela }}^{1}<G_{\text {min }}^{1}$ is met, then the green indication of phase 1 will remain $G_{\text {min }}^{1}-G_{\text {ela }}^{1}$ seconds. After $G_{\min }^{1}-G_{\text {ela }}^{1}$ seconds, the signal transformation mentioned above will be carried out on the basis of satisfying

$$
\begin{aligned}
& G_{\text {min }}^{1}-G_{\text {ela }}^{1}+t_{\text {switch }}+G_{\text {min }}^{2}+t_{\text {switch }}+\frac{q_{1} \times B}{v_{\text {reg }}}+t_{\text {safe }} \\
& \quad \leq \frac{L_{\mathrm{EV}-1}}{v_{\mathrm{EV}}} .
\end{aligned}
$$

It can be seen from (3) and (4) that, when $G_{\text {ela }}^{1}<G_{\text {min }}^{1}$, if (4) is satisfied then (3) is sure to be satisfied. Let $G_{\text {low }}$ denote the allowed lower bound of green light time that is smaller than $G_{\text {min }}^{1}$, and $G_{\text {short }}^{j}$ denote the shortened green light time of phase $j$. If (3) is satisfied while (4) is not satisfied, then $G_{\text {low }}$ is given. If $G_{\text {ela }}^{1} \geq G_{\text {low }}$, then $t_{E}(1)=t_{0}+G_{\text {min }}^{2}+2 t_{\text {switch }}$. If (3) is also not satisfied, then the green light time of pahse 2 will be shortened to $G_{\text {short }}^{2}$ to satisfy the following equation:

$$
t_{\text {switch }}+G_{\text {short }}^{2}+t_{\text {switch }}+\frac{q_{1} \times B}{v_{\text {reg }}}+t_{\text {safe }}=\frac{L_{\mathrm{EV}-1}}{v_{\mathrm{EV}}} .
$$

Thus we have

$$
G_{\text {short }}^{2}=\frac{L_{\mathrm{EV}-1}}{v_{\mathrm{EV}}}-\left(2 t_{\text {switch }}+\frac{q_{1} \times B}{v_{\text {reg }}}+t_{\text {safe }}\right) .
$$

If $G_{\text {short }}^{2}<G_{\text {low }}$, it shows that the green light time of phase 2 is very short and has little effect on alleviating traffic pressure and can lead to security problems. Therefore, $t_{E}(1)=t_{0}$ under this condition. Otherwise, the green light time of pahse 2 is equal to $G_{\text {short }}^{2}$, and $t_{E}(1)$ is equal to $t_{0}+G_{\text {short }}^{2}+t_{\text {switch. The }}$ real start time of green signal is $t_{E}(1)$.

(2) Phase 2 is the Current Phase of Intersection 1 at Time $t_{0}$. Calculate $G_{\text {ela }}^{2}$ firstly. If $G_{\text {ela }}^{2} \leq G_{\text {min }}^{2}$, it should be guaranteed generally that the green duration of phase 2 is $G_{\min }^{2}$ seconds and then changed to phase 1 after fixed yellow and red duration. However, it should be guaranteed that

$$
G_{\text {min }}^{2}-G_{\text {ela }}^{2}+t_{\text {switch }}+\frac{q_{1} \times B}{v_{\text {reg }}}+t_{\text {safe }} \leq \frac{L_{\mathrm{EV}-1}}{v_{\mathrm{EV}}} .
$$

Now, $t_{E}(1)$ is equal to $t_{0}+G_{\min }^{2}-G_{\text {ela }}^{2}+t_{\text {switch }}$. Otherwise, we have to check whether the following equation is satisfied:

$$
G_{\text {low }}-G_{\text {ela }}^{2}+t_{\text {switch }}+\frac{q_{1} \times B}{v_{\text {reg }}}+t_{\text {safe }} \leq \frac{L_{\mathrm{EV}-1}}{v_{\mathrm{EV}}} .
$$

If it is satisfied, then $t_{E}(1)$ is equal to $t_{0}+G_{\text {low }}-G_{\text {ela }}^{2}+t_{\text {switch }}$. If $G_{\text {ela }}^{2}>G_{\text {min }}^{2}$, then determine whether the following equation is satisfied:

$$
t_{\text {switch }}+\frac{q_{1} \times B}{v_{\text {reg }}}+t_{\text {safe }} \leq \frac{L_{\mathrm{EV}-1}}{v_{\mathrm{EV}}} .
$$

If it is satisfied, then $t_{E}(1)$ is equal to $t_{0}+t_{\text {switch }}$.

2.1.2. The Calculation of $t_{L}(1)$. Assume that $t_{\mathrm{arr}}^{i}$ denotes the time that EV arrive at intersection $i$ without reducing its speed; then we have

$$
t_{\mathrm{arr}}^{1}=t_{0}+\frac{L_{\mathrm{EV}-1}}{v_{\mathrm{EV}}} .
$$

Let $t_{\text {clear }}^{i}$ denote the time needed to clear the queues at intersection $i$ before $\mathrm{EV}$ arrives, then we have

$$
t_{\text {clear }}^{1}=\frac{q_{1} \times B}{v_{\text {reg }}} \text {. }
$$

The value of $t_{L}(1)$ can be described by the following equation:

$$
t_{L}(1)=t_{0}+\frac{L_{\mathrm{EV}-1}}{v_{\mathrm{EV}}}-\frac{q_{1} \times B}{v_{\text {reg }}}-t_{\text {safe }} .
$$

If the distance between EV detector and intersection 1 is far enough and queued vehicles are not too much, $t_{L}(1)$ is sure greater than or equal to $t_{E}(1)$. However, if the traffic is rather heavy and clearing queued vehicles takes a long time, $t_{L}(1)$ may be less than $t_{E}(1)$ at phase 2 . Under this condition, to ensure the smooth and safe passing of EV, the green time of phase 2 has to be sacrificed; namely, the green signal is converted to phase 1 after phase 2 has executed a short green time that is less than the minimum green time. Now, the value of $t_{E}(1)$ is $t_{0}+t_{\text {switch. If }} t_{L}(1)$ is still less than $t_{E}(1)$, then let $t_{L}(1)$ and $t_{E}(1)$ have the same value, say, $t_{0}+t_{\text {switch }}$. Now, it is unable to meet the requirement of EV's passing through the intersection without deceleration and stopping, and all that we can do is to minimize the waiting time of EV at intersection 1 . 


\subsection{Calculation of Time Parameters at Other Intersections}

2.2.1. The Calculation of $t_{L}(2)$. Let $t_{\text {pass }}$ be the time EV needs to pass through the intersection, $t_{\text {arr }}^{2}$ the time EV needs to arrive at the parking line of intersection 2 after passing through intersection 1 without stopping, $t_{\text {start }}^{i}$ the real start time of green signal of intersection $i$ along the evacuation direction of $E V$, and $Q_{i}$ and $D_{i}$ the number of vehicles arriving at intersection $i+1$ from intersection $i$ and vehicles departing from intersection $i$ in $t_{0}$ to $t_{\text {start }}^{i}$ time period, respectively. Then we have

$$
t_{\mathrm{arr}}^{2}=t_{0}+\frac{L_{\mathrm{EV}-2}}{v_{\mathrm{EV}}}+t_{\mathrm{pass}} .
$$

And the queuing vehicles $q_{2}^{\prime}$ at intersection 2 can be described as

$$
q_{2}^{\prime}=q_{2}+Q_{1}-D_{2}
$$

where $Q_{i}$ and $D_{i}$ are calculated according to the situation that the green light of intersection 1 starts at the latest-possible start time. Let $t_{\text {clear }}^{2}$ be the time needed for clearing $q_{2}^{\prime}$ before $\mathrm{EV}$ arrives; then we have

$$
t_{\text {clear }}^{2}=\frac{q_{2}^{\prime} \times B}{v_{\text {reg }}}=\frac{\left(q_{2}+Q_{1}-D_{2}\right) \times B}{v_{\text {reg }}} .
$$

We have the following equation to determine the latest start time of green signal at intersection 2 :

$$
\begin{aligned}
t_{L}(2)= & t_{\text {arr }}^{2}-t_{\text {clear }}^{2}-t_{\text {safe }} \\
= & t_{0}+\frac{L_{\mathrm{EV}-2}}{v_{\mathrm{EV}}}+t_{\text {pass }}-\frac{\left(q_{2}+Q_{1}-D_{2}\right) \times B}{v_{\text {reg }}} \\
& -t_{\text {safe }} .
\end{aligned}
$$

\subsubsection{The Calculation of $t_{E}(2)$}

(1) Phase 1 is the Current Phase of Intersection 2 at Time $t_{0}$. If the elapsed green time of phase 1 is $G_{\mathrm{ela}}^{1}, G_{\mathrm{ela}}^{1}+L_{\mathrm{EV}-2} / v_{\mathrm{EV}}+t_{\text {pass }}$ is the sum of the elapsed green time of phase 1 and the time $\mathrm{EV}$ spent before reaching intersection 2 . We can discuss the calculation of $t_{E}(2)$ in the following three cases.

Case 1.

$$
G_{\text {ela }}^{1}+\frac{L_{\mathrm{EV}-2}}{v_{\mathrm{EV}}}+t_{\text {pass }} \leq G_{\text {real }}^{1} .
$$

Case 2.

$$
G_{\text {real }}^{1}<G_{\text {ela }}^{1}+\frac{L_{\mathrm{EV}-2}}{v_{\mathrm{EV}}}+t_{\mathrm{pass}} \leq G_{\mathrm{max}}^{1}
$$

Case 3.

$$
G_{\mathrm{ela}}^{1}+\frac{L_{\mathrm{EV}-2}}{v_{\mathrm{EV}}}+t_{\text {pass }}>G_{\max }^{1}
$$

In Case 1 , there is no need to perform signal preemption at intersection 2 , and both $t_{E}(2)$ and the real start time of green signal are equal to $t_{0}$.

In Case 2, after both phase 1 and phase 2 have executed the minimum green time and then green indication goes back to phase 1 , we should check whether the following equation is satisfied:

$$
\begin{aligned}
& \left(G_{\text {min }}^{1}-G_{\text {ela }}^{1}\right)+2 t_{\text {switch }}+G_{\text {min }}^{2}+\frac{q_{2}^{\prime} \times B}{v_{\text {reg }}}+t_{\text {safe }} \\
& \quad \leq \frac{L_{\mathrm{EV}-2}}{v_{\mathrm{EV}}}+t_{\text {pass }},
\end{aligned}
$$

where $q_{2}^{\prime}$ is the queue length of intersection 2 at time $\left(G_{\min }^{1}-\right.$ $\left.G_{\text {ela }}^{1}\right)+2 t_{\text {switch }}+G_{\text {min }}^{2}$ and is calculated according to (14). In (14), $Q_{1}$ is calculated according to the situation that the green light of intersection 1 starts at the earliest-possible start time; $D_{2}$ is the number of vehicles passing through intersection 2 in the effective green duration of phase 1 in the period of $t_{0}$ to $t_{E}(2)$. If (20) is satisfied, then $t_{E}(2)$ is equal to $t_{0}+\left(G_{\min }^{1}-\right.$ $\left.G_{\text {ela }}^{1}\right)+2 t_{\text {switch }}+G_{\min }^{2}$; otherwise, $t_{E}(2)$ is equal to $t_{0}$.

In Case 3, we need to consider the restriction of the maximum green time. Let both phase 1 and phase 2 execute the maximum green time, and then let green indication go back to phase 1 . But we should check whether $q_{2}^{\prime}$ can be cleared before EV arrives, namely, whether the following equation is satisfied:

$$
\begin{aligned}
& \left(G_{\max }^{1}-G_{\text {ela }}^{1}\right)+2 t_{\text {switch }}+G_{\text {max }}^{2}+\frac{q_{2}^{\prime} \times B}{v_{\text {reg }}}+t_{\text {safe }} \\
& \quad \leq \frac{L_{\mathrm{EV}-2}}{v_{\mathrm{EV}}}+t_{\mathrm{pass}} .
\end{aligned}
$$

The calculation of $q_{2}^{\prime}$ is similar to Case 2. If (21) is satisfied, $t_{E}(2)$ is equal to $t_{0}+\left(G_{\max }^{1}-G_{\text {ela }}^{1}\right)+2 t_{\text {switch }}+G_{\max }^{2}$. Otherwise, let phase 1 and phase 2 execute the minimum green time and the maximum green time, respectively, and then let green indication go back to phase 1 . But the following equation should be satisfied:

$$
\begin{aligned}
& \left(G_{\text {min }}^{1}-G_{\text {ela }}^{1}\right)+2 t_{\text {switch }}+G_{\text {max }}^{2}+\frac{q_{2}^{\prime} \times B}{v_{\text {reg }}}+t_{\text {safe }} \\
& \quad \leq \frac{L_{\mathrm{EV}-2}}{v_{\mathrm{EV}}}+t_{\text {pass }} .
\end{aligned}
$$

Now, $t_{E}(2)$ is equal to $t_{0}+\left(G_{\min }^{1}-G_{\text {ela }}^{1}\right)+2 t_{\text {switch }}+G_{\text {max }}^{2}$.

If (22) is not satisfied, then both phase 1 and phase 2 will execute the minimum green time, and then green indication will go back to phase 1 . Let the cycle length be $C_{\max }$ when all of the two phases execute the maximum green time and $C_{\min }$ when all of the two phases execute the minimum green time.

(2) Phase 2 Is the Current Phase of Intersection 2 at Time $t_{0}$. If the elapsed green time of phase 2 is $G_{\text {ela }}^{2}$, we can discuss the calculation of $t_{E}(2)$ in the following four cases. 
Case 1.

$$
G_{\text {real }}^{2}-G_{\text {ela }}^{2}+t_{\text {switch }}+t_{\text {clear }}^{2}+t_{\text {safe }} \leq \frac{L_{\mathrm{EV}-2}}{v_{\mathrm{EV}}}+t_{\text {pass }} .
$$

Case 2.

$$
\begin{gathered}
G_{\text {min }}^{2}-G_{\text {ela }}^{2}+t_{\text {switch }}+t_{\text {clear }}^{2}+t_{\text {safe }} \leq \frac{L_{\mathrm{EV}-2}}{v_{\mathrm{EV}}}+t_{\text {pass }} \\
<G_{\text {real }}^{2}-G_{\text {ela }}^{2}+t_{\text {switch }}+t_{\text {clear }}^{2}+t_{\text {safe }}
\end{gathered}
$$

Case 3.

$$
\begin{gathered}
G_{\text {low }}-G_{\text {ela }}^{2}+t_{\text {switch }}+t_{\text {clear }}^{2}+t_{\text {safe }} \leq \frac{L_{\mathrm{EV}-2}}{v_{\mathrm{EV}}}+t_{\text {pass }} \\
<G_{\text {min }}^{2}-G_{\text {ela }}^{2}+t_{\text {switch }}+t_{\text {clear }}^{2}+t_{\text {safe }}
\end{gathered}
$$

Case 4.

$$
\frac{L_{\mathrm{EV}-2}}{v_{\mathrm{EV}}}+t_{\mathrm{pass}}<G_{\mathrm{low}}-G_{\text {ela }}^{2}+t_{\text {switch }}+t_{\text {clear }}^{2}+t_{\text {safe }}
$$

Case 1 corresponds to the situation that the time needed by green light of phase 1 turning green after the normal green duration of phase 2 and clearing the queue length of phase 1 is no more than the time spent by EV for arriving at intersection 2 ; then there is no need to perform signal preemption at intersection 2 and $t_{E}(2)$ is equal to $t_{0}+G_{\text {real }}^{2}-G_{\text {ela }}^{2}+t_{\text {switch }}$. Case 2 corresponds to the situation that the time needed by phase 1 turning green after the minimum green duration of phase 2 and clearing the queue length of phase 1 is no more than the time spent by EV for arriving at intersection 2 ; then signal preemption is necessary and $t_{E}(2)$ is equal to $t_{0}+G_{\min }^{2}-G_{\text {ela }}^{2}+t_{\text {switch. }}$ In Case 3, green light of phase 1 turns green after phase 2 has passed $G_{\text {low }}$ time, and now $t_{E}(2)$ is

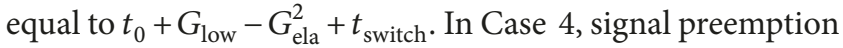
is necessary at intersection 2 , and the green duration of phase 2 has to be sacrificed; namely, the green signal has to turn to phase 1 immediately after detecting $E V$, and now $t_{E}(2)$ is equal to $t_{0}+G_{\text {ela }}^{2}+t_{\text {switch }}$.

The time parameters of the other intersections on the line are similar to those of intersection 2, which is no longer introduced here.

\section{Multi-Objective Optimization Model}

Let $n$ be the number of intersections on the evacuation route, $m$ the number of entrance sections on the cross road of evacuation route; $t_{\text {dep }}^{i}$ the time instant EV departing from intersection $i ; g_{\mathrm{eff}}^{i, j}, s_{i, j}^{y-z}$, and $S_{i, j}$ the effective green time of phase $j$ at intersection $i$, the average departure rate of vehicles from $y$ to $z$ directions on the entrance sections, and the saturation flow rate of all lanes on the entrance sections, respectively; and $A_{\mathrm{thr}}^{i, j}, A_{\mathrm{left}}^{i, j}$, and $A_{\text {right }}^{i, j}$ the ratio of straight, left turn and right turn of phase $j$ at intersection $i$, respectively. The multiobjective optimization model for $\mathrm{EV}$ signal preemption control based on route is

$\min f_{1}=\sum_{i=1}^{n}\left(t_{\text {start }}^{i}+t_{\text {clear }}^{i}+t_{\text {safe }}-t_{\text {arr }}^{i}\right) \times \delta_{i}$,

$\max f_{2}$

$$
\begin{aligned}
& =\sum_{i=1}^{n} g_{\mathrm{eff}}^{i, 2} \\
& \times\left[\left(s_{i, 2}^{N-S}+s_{i, 2}^{S-N}\right) \times A_{\mathrm{thr}}^{i, 2}+s_{i, 2}^{N-S} A_{\text {left }}^{i, 2}+s_{i, 2}^{S-N} A_{\text {right }}^{i, 2}\right], \\
& \text { s.t. } \quad t_{E}(i) \leq t_{\text {start }}^{i} \leq t_{L}(i) \quad i=1,2, \ldots, n, \\
& t_{\mathrm{arr}}^{i}=\frac{L_{\mathrm{EV}-i}}{v_{\mathrm{EV}}} \quad i=1,2, \ldots, n, \\
& t_{\text {clear }}^{i}=\frac{q_{i}^{\prime} \times B}{v_{\text {reg }}} \quad i=2, \ldots, n, \\
& q_{i}^{\prime} \\
& =q_{i}+\min \left\{q_{i-1}^{\prime} \times A_{\mathrm{thr}}^{i-1,1}, g_{\mathrm{eff}}^{i-1,1} \times S_{i-1, j} \times A_{\mathrm{thr}}^{i-1,1}\right\} \\
& +g_{\mathrm{eff}}^{i-1,2}\left(s_{i, 2}^{N-S} A_{\text {left }}^{i, 2}+s_{i, 2}^{S-N} A_{\text {right }}^{i, 2}\right) \\
& -\sum_{e=1}^{l} \min \left\{q_{e}^{\prime \prime}, g_{\mathrm{eff}}^{i, 1} \times A_{\mathrm{thr}}^{i, 1}\right\} \text {, } \\
& \delta_{i}=\left\{\begin{array}{ll}
0 & t_{\text {start }}^{i}+t_{\text {clear }}^{i}+t_{\text {safe }} \leq t_{\text {arr }}^{i} \\
1 & \text { otherwise }
\end{array} \quad i=2, \ldots, n,\right. \\
& t_{\text {safe }} \geq 2, \\
& t_{E}(i) \geq t_{0} \quad i=1,2, \ldots, n, \\
& t_{L}(i) \geq t_{0} \quad i=1,2, \ldots, n,
\end{aligned}
$$

where (27) and (28) are objective functions and represent minimizing the residence time of $\mathrm{EV}$ at all intersections and maximizing the number of social vehicles passing through all intersections of the evacuation route, respectively. The number of social vehicles includes the vehicles entering in the system from the cross road of evacuation route and passing through intersections and the vehicles passing through intersections in the direction of the evacuation route of EV. Equation (29) ensures that the real start time of green signal of intersection $i$ is between $t_{E}(i)$ and $t_{L}(i)$; (30) represents the arrival time of $\mathrm{EV}$ at intersection $i$; (31) represents the time needed to clear the queued vehicles before EV arrives; (32) denotes the calculation methd of the queued vehicles at intersection $i$ at the moment the green signal is starting, and $q^{\prime \prime}, e=1,2, \ldots, l$ represents the number of queued vehicles of phase 1 at the moment the green signal is starting in each signal cycle of intersection $i$ before $t_{\text {start }}^{i}$, and $l$ denotes the number of passed signal cycles; (33) represents the 01 variable of intersection $i$, which means that the waiting time of EV at intersection $i$ is 0 if the green signal is on at intersection $i$, and the queued vehicles have been cleared and security interval is satisfied, namely, $t_{\text {start }}^{i}+t_{\text {clear }}^{i}+t_{\text {safe }} \leq$ $t_{\text {arr }}^{i}$. Otherwise, the EV has to wait in line, and the waiting 
time is euqual to $t_{\text {start }}^{i}+t_{\text {clear }}^{i}+t_{\text {safe }}-t_{\text {arr }}^{i}$; (34) shows the lower limit of the value of $t_{\text {safe }}$; (35) and (36) represent the relationship between $t_{E}(i), t_{L}(i)$, and $t_{0}$. Variable $g_{\text {eff }}^{i, 2}$ will take different values according to different $t_{0}$ and $t_{\text {start }}^{i}$. The value of $g_{\text {eff }}^{1,2}$ and $g_{\text {eff }}^{2,2}$ can be calculated by (3)-(9) and (10)-(16) and (20)-(22), respectively, and the calculation method of $g_{\mathrm{eff}}^{i, 2}, i=3,4, \ldots, n$ is similar to $g_{\mathrm{eff}}^{2,2}$.

\section{Design of Solving Algorithm}

At present, the evolutionary algorithm is the main algorithm for solving multiobjective programming problems. Owing to the advantages of fast convergence speed and easy implementation, the particle swarm algorithm (PSO) has been successfully applied in many optimization problems [48-52]. In this paper, PSO is adopted to solve the given model.

4.1. Encoding and Particle Swarm Generation. Generate particle swarm $P$ with $N_{1}$ particles randomly, and each particle can be represented as $X=\left(x_{1}, x_{2}, \ldots, x_{n}\right)$, where $x_{i}, i=$ $1,2, \ldots, n$ denotes $t_{\text {start }}^{i}$ of intersection $i$ and $x_{i} \in\left[t_{E}(i), t_{L}(i)\right]$. If rand( ) represents a random number between 0 and 1 , then the specific method of producing $x_{i}, i=1,2, \ldots, n$ is defined as follows:

$$
x_{i}=\operatorname{int}\left[t_{E}(i)+\left(t_{L}(i)-t_{E}(i)\right) \times \operatorname{rand}()\right] \text {. }
$$

4.2. Density of Solutions. Since the density of nondominated solutions is inversely proportional to their diversity [48], to obtain uniformly distributed Pareto frontier, the diversity of solutions should be guaranteed; that is, the sparse solutions should be retained as many as possible. For this reason, the solution density is introduced in this paper. Suppose that there are $p$ objectives and there are $S$ solutions in dominant population $E$. If the solutions in $E$ are sorted in ascending order according to their fitness values and $f_{j}(i), j=1,2, \ldots, p ; i=1,2, \ldots, S$ represents the $i$ th solution when sorting by the $j$ th objective, then the solution density is defined as follows:

$$
\begin{gathered}
\sum_{j=1}^{p}\left[f_{j}(i+1)-f_{j}(i-1)\right]+\sum_{j=1}^{p}\left[f_{j}(i)-f_{j}(i-1)\right] \\
=\sum_{j=1}^{p} f_{j}(i+1)+\sum_{j=1}^{p} f_{j}(i)-2 \sum_{j=1}^{l} f_{j}(i-1) .
\end{gathered}
$$

4.3. Updating of Location and Velocity. In standard multiobjective PSO algorithm, location updating requires the inertia factor $w$ and learning factors $c_{1}$ and $c_{2}$. Since a larger $w$ is suitable for a wide range exploration of solution space, while a smaller $w$ is suitable for detailed search on the target, the learning factors are adopted to balance the cognitive abilities of individuals and groups. To achieve better search and balance, some scholars put forward the idea that inertia factor and learning factors can change dynamically [49]. In this paper, inertia factor and learning factors are changed as follows:

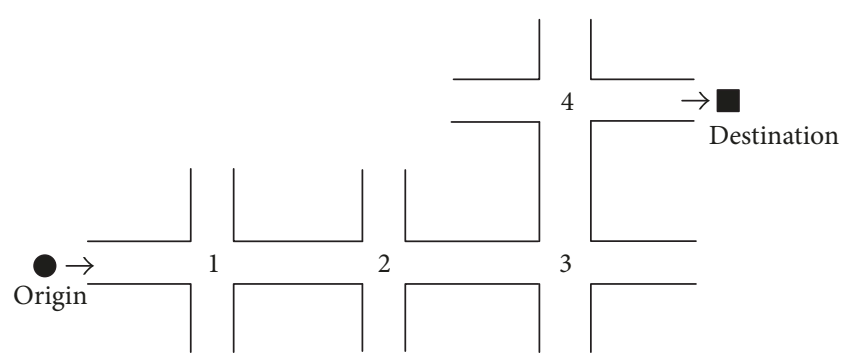

FIGURE 3: Intersections on evacutation route.

$$
\begin{aligned}
& w(k)=\left(w_{f}-w_{s}\right) \times \frac{\operatorname{Max}-k}{\operatorname{Max}}+w_{s}, \\
& c_{1}(k)=\left(c_{1 f}-c_{1 s}\right) \times \frac{\operatorname{Max}-k}{\operatorname{Max}}+c_{1 s}, \\
& c_{2}(k)=\left(c_{2 f}-c_{2 s}\right) \times \frac{\operatorname{Max}-k}{\operatorname{Max}}+c_{2 s},
\end{aligned}
$$

where $k, \operatorname{Max}, w_{s}$, and $w_{f}$ represent current iterations, maximum iterations, the initial value of inertia weight, and the final value of inertia weight, respectively; $c_{1 s}, c_{2 s}$ represent the initial value of $c_{1}$ and $c_{2}$, respectively; $c_{1 f}, c_{2 f}$ represent the final value of $c_{1}$ and $c_{2}$, respectively.

4.4. Mutation. In order to prevent the algorithm from falling into local optimum, mutation operator is introduced. Let $O$ be the mutation probability; if $k<\operatorname{Max} \times O$, then $\operatorname{Max} \times O-$ $k$ particles are selected from $P$ and mutate according to the following equation:

$$
x(i)=x(i)+\operatorname{rand}() \times \operatorname{int}\left(\frac{t_{\mathrm{L}}(i)-t_{\mathrm{E}}(i)}{2}-\frac{x(i)}{2}\right) .
$$

This will not only ensure the diversification of particles but also ensure the feasibility of particles after mutation.

\section{Simulation Analysis}

Assume that the evacuation route is composed of 4 intersections, as shown in Figure 3. The evacuation direction of EV is from west to east. It will pass through intersection 1 and intersection 2 straight ahead, turn left at intersection 3 , and turn right at intersection 4 , and $L_{\mathrm{EV}-1}=400 \mathrm{~m}, L_{\mathrm{EV}-2}=$ $850 \mathrm{~m}, L_{\mathrm{EV}-3}=1350 \mathrm{~m}, L_{\mathrm{EV}-4}=1850 \mathrm{~m}, v_{\mathrm{EV}}=45 \mathrm{~km} / \mathrm{h}$, $v_{\text {reg }}=45 \mathrm{~km} / \mathrm{h} ; d_{i 1}=d_{i 2}=28 \mathrm{~s}, C_{i}=56 \mathrm{~s}, i=1,2,3,4$, $C_{\max }=120 \mathrm{~s}, C_{\min }=30 \mathrm{~s} ; w s_{1}=0 \mathrm{~s}, w s_{2}=30 \mathrm{~s}, w s_{3}=60 \mathrm{~s}$, $w s_{4}=90 \mathrm{~s} ; t_{\text {switch }}=3 \mathrm{~s}, t_{\text {pass }}=2 \mathrm{~s}, B=7 \mathrm{~m} ; G_{\text {min }}^{1}=G_{\min }^{2}=$ $15 \mathrm{~s}, G_{\max }^{1}=G_{\max }^{2}=60 \mathrm{~s}, G_{\text {real }}^{1}=G_{\text {real }}^{2}=25 \mathrm{~s}, G_{\text {low }}=10 \mathrm{~s}$, $G_{\text {short }}^{j}=10 \mathrm{~s}$.

In south-northward direction and west-eastward direction, the ratio of straight, right turn, and left turn is $0.7,0.2$, and 0.1 , respectively. And it is $0.6,0.3$, and 0.1 respectively, in north-southward direction. In the particle swarm algorithm, $N_{1}=100, \operatorname{Max}=1000$, and $O=0.1$. The size of external dominance population is 100 , and $w_{s}=0.4, w_{f}=0.9, c_{1 f}=$ $1, c_{2 f}=2, c_{1 s}=2, c_{2 s}=1$. 
TABLE 1: The Pareto solution set of of EV when $t_{0}$ is 9 o'clock.

\begin{tabular}{|c|c|c|c|c|c|c|}
\hline \multirow{2}{*}{ Number } & \multicolumn{4}{|c|}{ The real start time of green signal(s) } & \multirow{2}{*}{$f_{1}(\mathrm{~s})$} & \multirow{2}{*}{$f_{2}(\mathrm{pcu})$} \\
\hline & Intersection 1 & Intersection 2 & Intersection 3 & Intersection 4 & & \\
\hline (1) & 10 & 33 & 53 & 81 & 0 & 112.1 \\
\hline (2) & 10 & 34 & 54 & 81 & 2.3 & 113.6 \\
\hline (3) & 10 & 34 & 55 & 82 & 2.4 & 114.8 \\
\hline (4) & 11 & 34 & 55 & 83 & 3.9 & 116.6 \\
\hline (5) & 12 & 34 & 56 & 83 & 4.7 & 118.7 \\
\hline$(6)$ & 13 & 34 & 58 & 84 & 4.8 & 120.4 \\
\hline
\end{tabular}

TABle 2: The Pareto solution set of EV when $t_{0}$ is 10 oclock.

\begin{tabular}{|c|c|c|c|c|c|c|}
\hline \multirow{2}{*}{ Number } & \multicolumn{4}{|c|}{ The real start time of green signal(s) } & \multirow[b]{2}{*}{$f_{1}(\mathrm{~s})$} & \multirow{2}{*}{$f_{2}(\mathrm{pcu})$} \\
\hline & Intersection 1 & Intersection 2 & Intersection 3 & Intersection 4 & & \\
\hline (1) & 10 & 31 & 56 & 82 & 0 & 119.7 \\
\hline (2) & 11 & 33 & 58 & 83 & 2.9 & 119.9 \\
\hline (3) & 11 & 34 & 57 & 83 & 3.2 & 122.4 \\
\hline (4) & 12 & 34 & 58 & 83 & 4.9 & 122.7 \\
\hline$(5)$ & 13 & 34 & 57 & 83 & 5.5 & 123.5 \\
\hline
\end{tabular}

Assume that the number of vehicles waiting to pass through each intersection at time $t_{0}$ is $q_{1}=10, q_{2}=5, q_{3}=$ 6 , and $q_{4}=8$, respectively, and $q_{i \mathrm{~N}}=q_{i \mathrm{~s}}=10, i=1,2$, $q_{3 \mathrm{~S}}=q_{3 \mathrm{E}}=10$, and $q_{4 \mathrm{~N}}=q_{4 \mathrm{~W}}=10$. At each intersection, the arrival rate from south to north is $1080-1440 \mathrm{pcu} / \mathrm{h}$ and the average departure rate is $1440 \mathrm{pcu} / \mathrm{h}$, the arrival rate from east to west is $1440-1800 \mathrm{pcu} / \mathrm{h}$, and the saturation flow rate is $1800 \mathrm{pcu} / \mathrm{h}$. Assume that $t_{0}$ is 9 oclock and 10 oclock, respectively. The Pareto solution set obtained by using C\# for simulation calculation is shown in Tables 1 and 2. The data corresponding to each intersection indicates how many seconds after 9 o'clock or 10 o'clock will each intersection start the green signal; $f_{1}$ and $f_{2}$ are the residence time of $\mathrm{EV}$ at all the intersections and the number of vehicles passing through the system, respectively.

Assume that the number of vehicles waiting to pass through each intersection at time $t_{0}$ is $q_{i}=20, i=1,2,3,4$, and $q_{i \mathrm{~N}}=q_{i \mathrm{~S}}=10, i=1,2, q_{3 \mathrm{~S}}=q_{3 \mathrm{E}}=10, q_{4 \mathrm{~N}}=$ $q_{4 \mathrm{~W}}=10$. Traffic flow from north to south and from west to east is the key traffic flow. At each intersection, the arrival rate from north to south is $1400-1600 \mathrm{pcu} / \mathrm{h}$ and the average departure rate is $1440 \mathrm{pcu} / \mathrm{h}$; the arrival rate from west to east is $1800-1900 \mathrm{pcu} / \mathrm{h}$ and the saturation flow rate is $1800 \mathrm{pcu} / \mathrm{h}$; the arrival rate in other directions remain unchanged. If $t_{0}$ is 9 o'clock and 10 o'clock, respectively, the Pareto solution set is shown in Tables 3 and 4.

To verify the control effect of the signal preemption control method presented in this paper under the condition of much larger traffic volume, for example, in the moring peak or the evening peak period, we assume that $t_{0}$ is 8 o'clock, namely, in the moring peak period, and $q_{i}=20, i=1,2,3,4$, $q_{i \mathrm{~N}}=q_{i \mathrm{~S}}=15, i=1,2, q_{3 \mathrm{~S}}=q_{3 \mathrm{E}}=15, q_{4 \mathrm{~N}}=q_{4 \mathrm{~W}}=15$. At each intersection, the arrival rate from north to south and from west to east is $1800-1900 \mathrm{pcu} / \mathrm{h}$ and $2200-2400 \mathrm{pcu} / \mathrm{h}$, respectively. The saturation flow rate is $1800 \mathrm{pcu} / \mathrm{h}$ and the

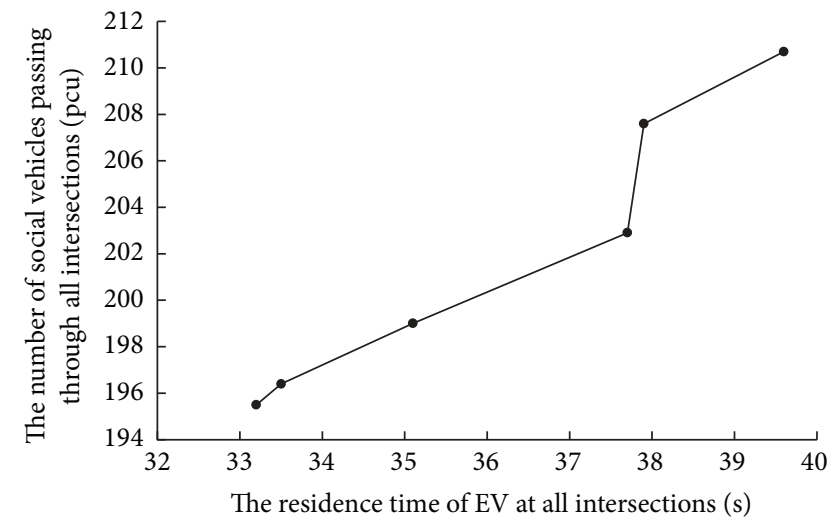

Figure 4: The Pareto optimal set.

arrival rate in other directions remain unchanged. The Pareto optimal set is shown in Figure 4.

We can see from the simulation results shown in Tables 1 and 2 that, for each solution in the Pareto solution set, the variation law of the two optimization objectives is that with the reduction of one objective; the other objective is reduced simultaneously, which implies that the decrease in the residence time of $\mathrm{EV}$ at intersections is at the expense of the reduction of the number of vehicles passing through the system. It also can be seen from the simulation results shown in Tables 3 and 4 that, at the moment the EV is detected, if there are many queued vehicles at each intersections and the traffic volume of key traffic flow exceeds the amount of traffic that the system can release, the control method can still guarantee the EV to pass through the intersection at a faster speed, and more social vehicles can pass through the system. The results shown in Figure 4 also indicate that the signal preemption control method is effective when the 
TABLE 3: The Pareto solution set for an increasing arrival rate when $t_{0}$ is 9 oclock.

\begin{tabular}{|c|c|c|c|c|c|c|}
\hline \multirow{2}{*}{ Number } & \multicolumn{4}{|c|}{ The real start time of green signal(s) } & \multirow{2}{*}{$f_{1}(\mathrm{~s})$} & \multirow{2}{*}{$f_{2}(\mathrm{pcu})$} \\
\hline & Intersection 1 & Intersection 2 & Intersection 3 & Intersection 4 & & \\
\hline (1) & 2 & 46 & 73 & 108 & 29.4 & 165.7 \\
\hline (2) & 2 & 45 & 75 & 108 & 29.5 & 167.1 \\
\hline (3) & 0 & 45 & 76 & 114 & 33.2 & 171.2 \\
\hline (4) & 1 & 43 & 76 & 111 & 36.7 & 175.4 \\
\hline (5) & 1 & 44 & 80 & 116 & 37.9 & 183.5 \\
\hline (6) & 4 & 44 & 80 & 111 & 38.4 & 184.4 \\
\hline$(7)$ & 7 & 43 & 82 & 123 & 38.4 & 186.9 \\
\hline (8) & 4 & 43 & 80 & 117 & 39.3 & 189.1 \\
\hline (9) & 7 & 43 & 84 & 119 & 39.9 & 193.6 \\
\hline
\end{tabular}

TABLE 4: The Pareto solution set for an increasing arrival rate when $t_{0}$ is 10 o'clock.

\begin{tabular}{|c|c|c|c|c|c|c|}
\hline \multirow{2}{*}{ Number } & \multicolumn{4}{|c|}{ The real start time of green signal(s) } & \multirow{2}{*}{$f_{1}(\mathrm{~s})$} & \multirow{2}{*}{$f_{2}(\mathrm{pcu})$} \\
\hline & Intersection 1 & Intersection 2 & Intersection 3 & Intersection 4 & & \\
\hline (1) & 11 & 43 & 79 & 110 & 27.2 & 169.9 \\
\hline (2) & 10 & 45 & 80 & 108 & 29.4 & 174.7 \\
\hline (3) & 4 & 33 & 76 & 106 & 29.7 & 180.2 \\
\hline (4) & 2 & 34 & 73 & 111 & 30.1 & 183.8 \\
\hline (5) & 6 & 46 & 79 & 116 & 31.8 & 185.7 \\
\hline (6) & 4 & 35 & 71 & 107 & 36.6 & 187.0 \\
\hline (7) & 12 & 43 & 77 & 108 & 38.3 & 189.2 \\
\hline
\end{tabular}

arrival rate are much larger in the morning peak period. Simulation results under different traffic states show that the signal preemption control method presented in this paper can reduce the delay of $\mathrm{EV}$ at intersections and lessen the impact of EV on social vehicles.

When multiple EVs arrive at the same time, the signal priority control method proposed in this paper is also applicable. If the arrival interval of adjacent EV is relatively long, it should be regarded as two signal priority processes. Now, after the front EV has passed through the intersection, we must consider signal transiton from priority signal to normal signal firstly and then adopt the method mentioned above to control the following EV.

\section{Conclusion}

In order to overcome the disadvantages of signal preemption control method from intersection to intersection, we study the dynamic signal preemption control method based on route for EV in this paper. Firstly, a single intersection is taken as the research object to determine the earliest-possible start time and the latest-possible start time of each intersection in evacuation direction of EV on the selected route. Then, considering the delay of EV at intersections and the effect of EV to social vehicles comprehensively, to ensure that EV can pass through the intersections without reducing speed and without stopping as far as possible and the impact on social vehicles can be minimal, a multiobjective programming model is established and the solving algorithm is designed. Simulation calculations, considering EV is detected at different times and different traffic states, are carried out, and the corresponding Pareto sets are given. The result shows that, by adopting this method, we can get more reasonable start time of green light at each intersection, reduce the delay of EV at intersections, and improve the efficiency of evacuation. Determine how the signal should be converted to the normal signal when signal preemption has been finished according to the signal timing, the traffic flow characteristics, and other parameters; namely, the signal transition strategy is the next focus of the study.

\section{Conflicts of Interest}

The authors declare that they have no conflicts of interest.

\section{Acknowledgments}

This research is supported by National Nature Science Foundation of China (nos. 61563029, 71671079, 71361018, and 71571090).

\section{References}

[1] Y.-S. Huang, Y.-S. Weng, and M.-C. Zhou, "Design of traffic safety control systems for emergency vehicle preemption using timed petri nets," IEEE Transactions on Intelligent Transportation Systems, vol. 16, no. 4, pp. 2113-2120, 2015.

[2] M. B. Younes and A. Boukerche, "An efficient dynamic traffic light scheduling algorithm considering emergency vehicles for intelligent transportation systems," Wireless Networks, pp. 1-13, 2017. 
[3] J. F. Paniati and M. Amoni, Traffic signal preemption for emergency vehicle: a cross-cutting study, US Federal Highway Administration, 2006.

[4] P. B. Mirchandani and D. E. Lucas, "Integrated transit priority and rail/emergency preemption in real-time traffic adaptive signal control," Journal of Intelligent Transportation Systems: Technology, Planning, and Operations, vol. 8, no. 2, pp. 101-115, 2004.

[5] H. H. Song, Study on multiple emergency vehicles signal priority control based on multi-agent approach, Harbin: Harbin Institute of Technology, Harbin Institute of Technology, Harbin, 2008.

[6] Z.-S. Yang, X.-M. Sun, and P.-C. Sun, "Signal priority control strategy and implementation for emergency vehicle at single intersection under traffic accidents," Jilin Daxue Xuebao (Gongxueban)/Journal of Jilin University (Engineering and Technology Edition), vol. 41, no. 3, pp. 640-644, 2011.

[7] C. Ma and J. X. Cui, "Multiple emergency vehicles signal priority control based on multi-agent approach," Journal of Transportation Systems Engineering and Information Technology, vol. 13, no. 1, pp. 57-62, 2013.

[8] J. Wang, W. Ma, and X. Yang, "Development of degree-ofpriority based control strategy for emergency vehicle preemption operation," Discrete Dynamics in Nature \& Society, vol. 2013, no. 1, pp. 215-227, 2013.

[9] X. Qin, Real time control and optimization of traffic signal timing transition for emergency vehicle preemption, University of Ottawa, Ottawa, Canada, 2005.

[10] F. A. Marcianò, G. Musolino, and A. Vitetta, "Signal setting optimization on urban road transport networks: The case of emergency evacuation," Safety Science, vol. 72, pp. 209-220, 2015.

[11] T. J. Kimpel, J. Strathman, R. L. Bertini, and S. Callas, "Analysis of transit signal priority using archived TriMet Bus Dispatch System data," Transportation Research Record, no. 1925, pp. 156166, 2005.

[12] X. Qin and A. M. Khan, "Control strategies of traffic signal timing transition for emergency vehicle preemption," Transportation Research Part C: Emerging Technologies, vol. 25, pp. $1-17,2012$.

[13] S. Shibuya, T. Yoshida, Z. Yamashiro, and M. Miyawaki, "Fast emergency vehicle preemption systems," Transportation Research Record Journal of the Transportation Research Board, vol. 1739, pp. 44-50, 2000.

[14] E. Kwon and S. Kim, Development of dynamic route clearance strategies for emergency vehicle operations, Phase I, University of Minnesota: Center for Transportation Studies, Center for Transportation Studies, 2003.

[15] H. K. Gedawy, M. B. Dias, and K. Harras, Dynamic path planning and traffic light coordination for emergency vehicle routing, Computer Science Department Carnegie Mellon University Pittsburgh, Pennsylvania, 2008.

[16] W. Kang, G. Xiong, Y. Lv, X. Dong, F. Zhu, and Q. Kong, “Traffic signal coordination for emergency vehicles," in Proceedings of the 2014 17th IEEE International Conference on Intelligent Transportation Systems, ITSC 2014, pp. 157-161, China, October 2014.

[17] S. Shekhar, K. S. Yang, V. M. V. Gunturi et al., "Experiences with evacuation route planning algorithms," International Journal of Geographical Information Science, vol. 26, no. 12, pp. 2253-2265, 2012.
[18] R. F. Kirby and R. B. Potts, "The minimum route problem for networks with turn penalties and prohibitions," Transportation Research, vol. 3, no. 3, pp. 397-408, 1969.

[19] E. H. E. Singer, "A method of finding cost-minimizing routes incorporating turn costs," Traffic, Engineering and Control, vol. 15, pp. 771-772, 1974.

[20] S. Namkoong and H. S. Mahmassani, "A note on least time path computation considering delays and prohibitions for intersection movements," Transportation Research Part B: Methodological, vol. 30, no. 5, pp. 359-367, 1996.

[21] X. D. Zou, S. F. Zheng, X. G. Ban, and X. M. Lian, "Optimal path algorithm for road Net with traffic capacity limits," Journal of Highway and Transportation Research and Development, vol. 19, pp. 82-84, 2002.

[22] Y. Z. Li and R. C. He, "Modeling for shortest path in traffic network with node limits," Operations Research and Management Science, vol. 14, pp. 40-43, 2005.

[23] F.-Y. Wang, F.-Q. Pan, L.-X. Zhang, and X.-D. Zou, "Optimal path algorithm of road network with traffic restriction," Journal of Traffic and Transportation Engineering, vol. 5, no. 1, pp. 92-95, 2005.

[24] T. J. Cova and J. P. Johnson, "A network flow model for lanebased evacuation routing," Transportation Research Part A: Policy and Practice, vol. 37, no. 7, pp. 579-604, 2003.

[25] R. Song, H. E. Shiwei, and L. Zhang, "Optimum transit operations during the emergency evacuations," Journal of Transportation Systems Engineering and Information Technology, vol. 9, no. 6, pp. 154-160, 2009.

[26] X. Chen and F. B. Zhan, "Agent-based modeling and simulation of urban evacuation: relative effectiveness of simultaneous and staged evacuation strategies," Agent-Based Modeling and Simulation, pp. 78-96, 2014.

[27] P. Murray-Tuite and B. Wolshon, "Evacuation transportation modeling: an overview of research, development, and practice," Transportation Research Part C: Emerging Technologies, vol. 27, pp. 25-45, 2013.

[28] E. Urbina and B. Wolshon, "National review of hurricane evacuation plans and policies: a comparison and contrast of state practices," Transportation Research Part A: Policy and Practice, vol. 37, no. 3, pp. 257-275, 2003.

[29] S. A. Parr and E. Kaisar, "Critical intersection signal optimization during urban evacuation utilizing dynamic programming," Journal of Transportation Safety \& Security, vol. 3, no. 1, pp. 5976, 2011.

[30] V. Campos, R. Bandeira, and A. Bandeira, "A Method for Evacuation Route Planning in Disaster Situations," Procedia Social and Behavioral Sciences, vol. 54, pp. 503-512, 2012.

[31] M. Gao and G. He, "Optimization of evacuation routes considering movements at intersections," Tumu Gongcheng Xuebao/China Civil Engineering Journal, vol. 40, no. 6, pp. 80-83, 2007.

[32] H. B. Mu, J. N. Yu, and L. Z. Liu, "Evacuation Routes Optimization with Effects of Traffic Light at Intersections," Journal of Transportation Systems Engineering \& Information Technology, vol. 11, no. 3, pp. 76-82, 2011.

[33] L. G. Chalmet, R. L. Francis, and P. B. Saunders, "NETWORK MODELS FOR BUILDING EVACUATION.," Management Science, vol. 28, no. 1, pp. 86-105, 1982.

[34] W. L. Wilkinson, "An algorithm for universal maximal dynamic flows in a network," Operations Research, vol. 19, pp. 1602-1612, 1971. 
[35] E. Minieka, "Maximal, lexicographic, and dynamic network flows," Operations Research, vol. 21, pp. 517-527, 1973.

[36] A. Stepanov and J. M. Smith, "Multi-objective evacuation routing in transportation networks," European Journal of Operational Research, vol. 198, no. 2, pp. 435-446, 2009.

[37] V. Bayram, B. Ç. Tansel, and H. Yaman, "Compromising system and user interests in shelter location and evacuation planning," Transportation Research Part B: Methodological, vol. 72, pp. 146163, 2015.

[38] N. Nassir, M. Hickman, H. Zheng, and Y.-C. Chiu, "Network flow solution method for optimal evacuation traffic routing and signal control with nonuniform threat," Transportation Research Record, vol. 2459, pp. 54-62, 2014.

[39] R. L. Church and R. M. Sexton, Modeling small area evacuation: Can existing transportation infrastructure impede public safety? Caltrans Testbed Center for Interoperability Task Order 3021 Report, Vehicle Intelligence and Transportation Analysis Laboratory, University of California, Santa Barbara, CA, USA, 2002.

[40] M. Ben-Akiva, D. Mcfadden, K. Train, J. Walker, C. Bhat, and M. Bierlaire, Development of a deployable real-time dynamic traffic assignment system: Dynamit and Dynamit-p users guide, Massachusetts Institute of Technology, Intelligent Transportation Systems Program, Cambridge, 2002.

[41] Q. Lu, Y. Huang, and S. Shekhar, "Evacuation Planning: A Capacity Constrained Routing Approach," in Intelligence and Security Informatics, vol. 2665 of Lecture Notes in Computer Science, pp. 111-125, Springer Berlin Heidelberg, Berlin, Heidelberg, 2003.

[42] Q. Lu, B. George, and S. Shekhar, "Capacity Constrained Routing Algorithms for Evacuation Planning: A Summary of Results," in Advances in Spatial and Temporal Databases, vol. 3633 of Lecture Notes in Computer Science, pp. 291-307, Springer Berlin Heidelberg, Berlin, Heidelberg, 2005.

[43] Q. Lu, B. George, and S. Shekhar, "Evacuation route planning: a case study in semantic computing," International Journal of Semantic Computing, vol. 1, no. 2, pp. 249-303, 2007.

[44] K. Shahabi and J. P. Wilson, "CASPER: Intelligent capacityaware evacuation routing," Computers, Environment and Urban Systems, vol. 46, pp. 12-24, 2014.

[45] E. Pourrahmani, M. R. Delavar, P. Pahlavani, and M. A. Mostafavi, "Dynamic evacuation routing plan after an earthquake," Natural Hazards Review, vol. 16, no. 4, Article ID 04015006, 2015.

[46] K. Shahabi and J. P. Wilson, "Scalable evacuation routing in a dynamic environment," Computers Environment Urban Systems, vol. 67, pp. 29-40, 2018.

[47] M. Zeng and C. Wang, "Evacuation route planning algorithm: Longer route preferential," Lecture Notes in Computer Science (including subseries Lecture Notes in Artificial Intelligence and Lecture Notes in Bioinformatics): Preface, vol. 5551, no. 1, pp. 1062-1071, 2009.

[48] D. F. Wang, L. Meng, and W. J. Zhao, "Improved bare bones particle swarm optimization with adaptive search center," Chinese Journal of Computers. Jisuanji Xuebao, vol. 39, no. 12, pp. 26522667, 2016.

[49] C. A. C. Coello, G. T. Pulido, and M. S. Lechuga, "Handling multiple objectives with particle swarm optimization," IEEE Transactions on Evolutionary Computation, vol. 8, no. 3, pp. 256279, 2004.
[50] B. Wei, Y. X. Li, X. Xu, and D. C. Shen, "Partical swarm optimization algorithm based on stable strategy," Computer Science, vol. 38, no. 12, pp. 221-223, 2011.

[51] H. Wang and F. Qian, "Improved PSO-based multi-objective optimization by crowding with mutation and particle swarm optimization dynamic changing," Kongzhi yu Juece/Control and Decision, vol. 23, no. 11, pp. 1238-1248, 2008.

[52] A. Ratnaweera, S. K. Halgamuge, and H. C. Watson, "Selforganizing hierarchical particle swarm optimizer with timevarying acceleration coefficients," IEEE Transactions on Evolutionary Computation, vol. 8, no. 3, pp. 240-255, 2004. 


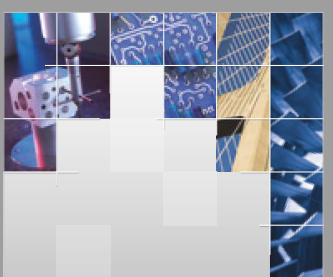

\section{Enfincering}
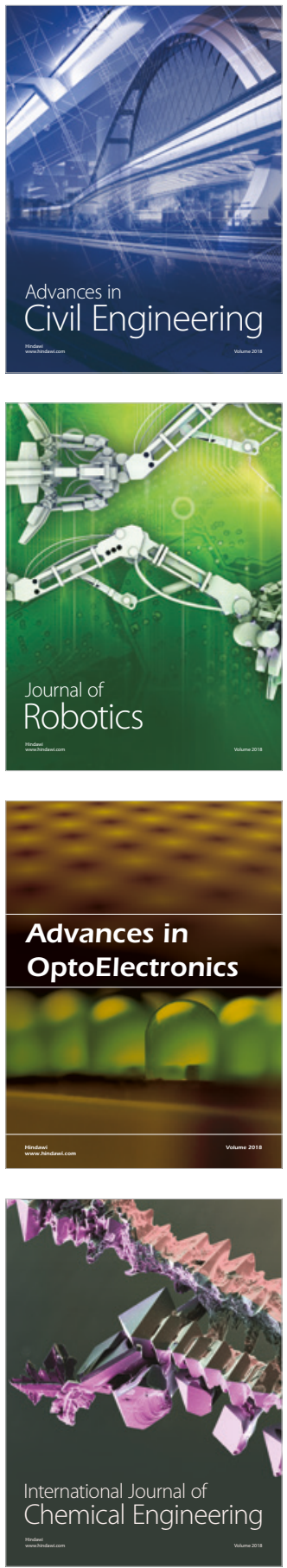

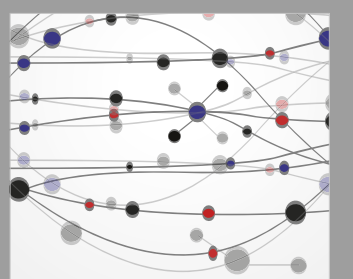

\section{Rotating \\ Machinery}

The Scientific World Journal

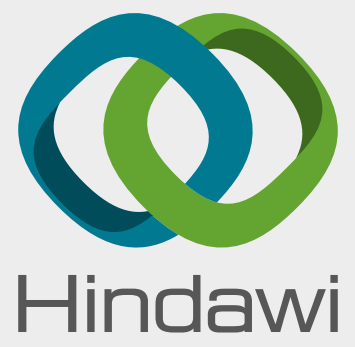

Submit your manuscripts at

www.hindawi.com
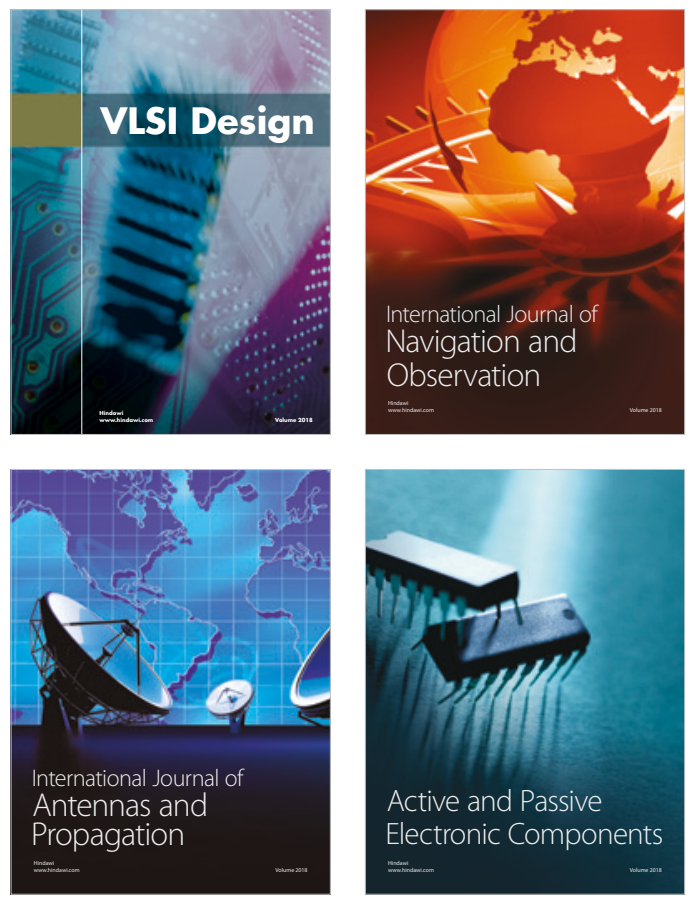
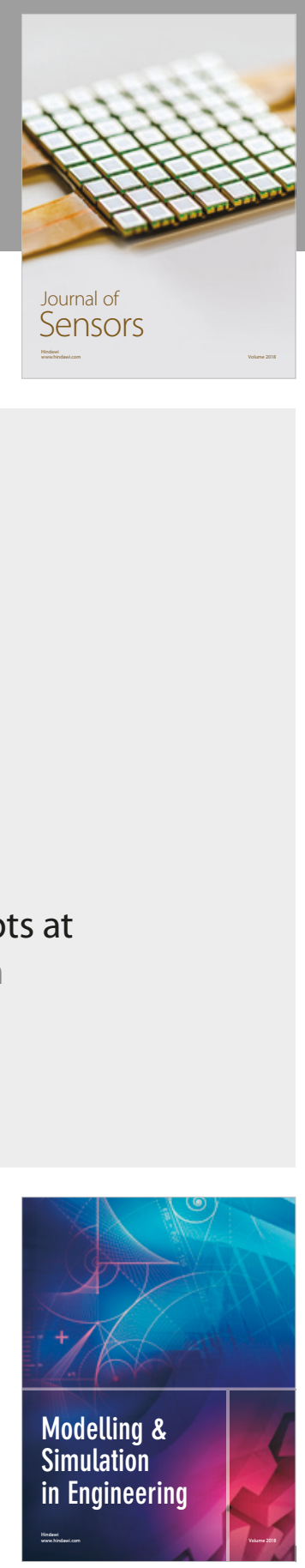

\section{Advances \\ Multimedia}
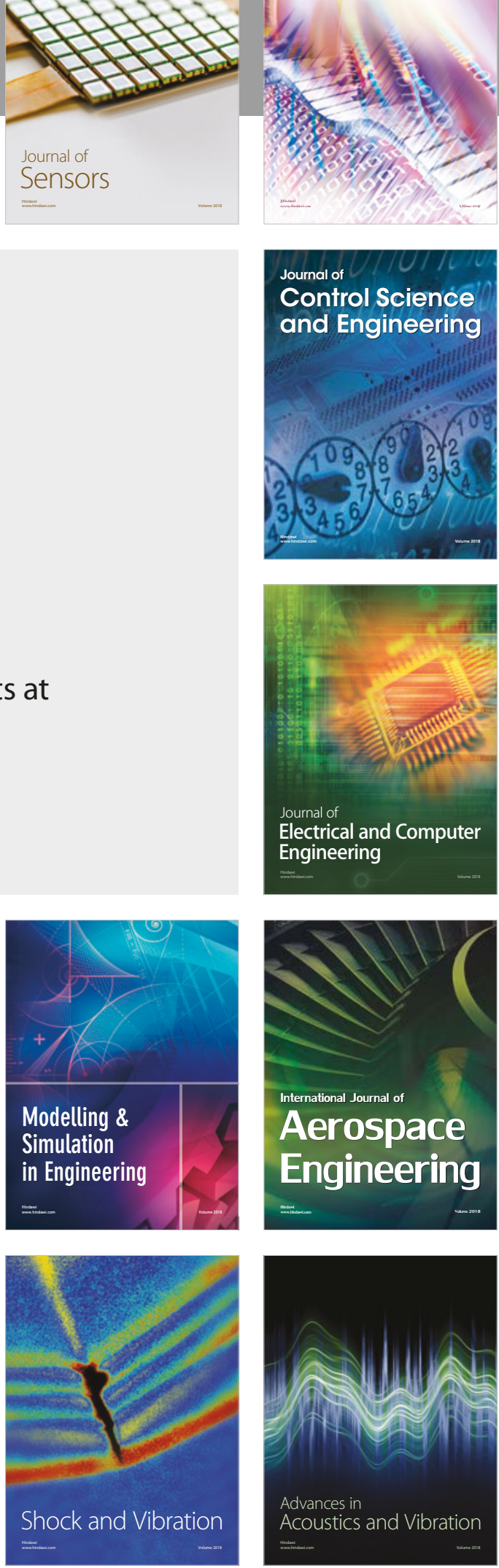